\title{
Daily Heart Rate Variability before and after Concussion in an American College Football Player
}

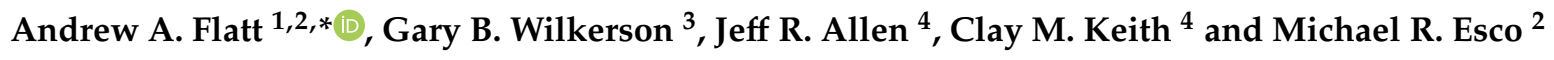 \\ 1 Georgia Southern University, Department of Health Sciences and Kinesiology, Biodynamics and Human \\ Performance Center, 11935 Abercorn, St. Savannah, GA 31419, USA \\ 2 Department of Kinesiology, Exercise Physiology Laboratory, University of Alabama, Tuscaloosa, AL 35487, \\ USA; mresco@ua.edu \\ 3 Graduate Athletic Training Program, University of Tennessee at Chattanooga, Chattanooga, TN 37403, USA; \\ gary-wilkerson@utc.edu \\ 4 Department of Athletics, Sports Medicine, University of Alabama, Tuscaloosa, AL 35487, USA; \\ jallen@ia.ua.edu (J.R.A.); ckeith@ia.ua.edu (C.M.K.) \\ * Correspondence: aflatt@georgiasouthern.edu; Tel.: +1-912-344-3317
}

Received: 21 March 2019; Accepted: 25 April 2019; Published: 27 April 2019

check for updates

\begin{abstract}
This case report demonstrates the effects of sport-related concussion (SRC) on heart rate variability (HRV) in an American college football player. Daily measures of resting, ultra-short natural logarithm of the root mean square of successive differences (LnRMSSD), subjective wellbeing, and Player Load were obtained each training day throughout a 4-week spring camp and 4 weeks of preseason training. SRC occurred within the first 2 weeks of the preseason. During spring camp and preseason pre-SRC, the athlete demonstrated minimal day-to-day fluctuations in LnRMSSD, which increased post-SRC (LnRMSSD coefficient of variation pre-SRC $\leq 3.1 \%$, post-SRC $=5.8 \%$ ). Moderate decrements in daily-averaged LnRMSSD were observed post-SRC relative to pre-SRC (Effect Size $\pm 90 \%$ Confidence Interval $=-1.12 \pm 0.80$ ), and the 7 -day rolling average fell below the smallest worthwhile change for the remainder of the preseason. LnRMSSD responses to SRC appeared similar to trends associated with stress and training fatigue. Therefore, performance and sports medicine staff should maintain regular communication regarding player injury and fatigue status so that HRV can be interpreted in the appropriate context. Detection and monitoring of autonomic dysregulation post-SRC may require near-daily assessment, as LnRMSSD showed greater daily fluctuations rather than chronic suppression following the head injury.
\end{abstract}

Keywords: autonomic; cardiac-parasympathetic; traumatic brain injury; sports medicine; sports science

\section{Introduction}

Player monitoring strategies and data analysis techniques in collegiate football are generally managed by performance staff. Microsensor-derived training load, subjective well-being, and ultra-short heart rate variability (HRV) represent a few of the measures currently being used by American college football teams in an effort to optimize player health and performance [1-4]. However, effective use of player monitoring tools such as HRV requires interpretation of the data in appropriate context given its sensitivity to a variety of physiological, psychological, and environmental factors [5].

High speed collisions put players at risk for sustaining sport-related concussion (SRC), which accounts for $8 \%$ of all reported American football injuries [6]. Emerging evidence from advanced neuroimaging and neurophysiological studies strongly suggest that SRC can have long-lasting adverse effects on brain function $[7,8]$. Interrelationships among neurometabolic, hormonal, and 
mechanical factors present an exceedingly complex challenge to clinicians who must make decisions in the management of highly heterogeneous manifestations of SRC [9]. Symptoms are poor indicators of neurological recovery, and standard clinical tests have been found inadequate for detection of subtle abnormalities that can persist beyond return to sport participation $[10,11]$. A number of published reports have established HRV as a reliable marker of autonomic dysregulation after SRC [10,12,13], potentially confounding interpretation of fatigue-induced alterations among collision-sport athletes [1,2,14-16]. Daily HRV averaged over a period of time (e.g., 1-2 weeks) reflects mean parasympathetic activity while its coefficient of variation $(\mathrm{CV})$ reflects the magnitude of daily HRV fluctuations. Reductions in mean HRV and increases in its CV are commonly observed in fatigued team-sport and anaerobic-event athletes $[15,17,18]$, reflecting withdrawn and unstable cardiac-parasympathetic activity. Thus, alterations in HRV patterns induced by training fatigue or SRC may be indistinguishable in the absence of added contextual information.

Inter-individual differences and lability in HRV make the availability of pre-injury baseline data essential for identification of persisting SRC effects, which has been presented in only one recent report [19]. Evidence of an association between SRC and altered HRV has been derived from time-consuming measurement protocols and sophisticated instrumentation, limiting its wide-spread implementation among sports teams. Further investigation into the usefulness of more convenient and time-efficient procedures for obtaining HRV that are currently being used for monitoring training adaptations in collision-sport athletes is needed [1,2,14-16]. Moreover, given that members of the performance staff (e.g., strength and conditioning coaches) are often managing the HRV data of players, a greater understanding of the effects of SRC on HRV is critical to recognize responses that may require attention from the sports medicine staff. Thus, the purpose of this case presentation is to report longitudinal changes in HRV from measurements acquired before and after the occurrence of SRC in a college football player.

\section{Materials and Methods}

\subsection{Participant}

This observational case report reviews data acquired from a Division-1 college football player (non-lineman) in the weeks before and after a SRC. Informed consent and ethical approval (6253) was obtained to use the acquired data for research purposes.

\subsection{Procedures}

\subsubsection{Observation Period}

The player was monitored throughout spring camp (mid-March-mid-April) and preseason football training (August-September). Resting HRV, subjective indicators of recovery status, and Player Load were obtained each training day throughout the observation periods. Variables were compared across three time-points as follows: (1) spring camp, (2) pre-season, pre-SRC (Pre-SRC) and (3) pre-season, post-SRC (Post-SRC). SRC-indicators were collected following SRC-occurrence and were compared to pre-existing baseline values.

\subsubsection{Heart Rate Variability}

Resting-HRV was obtained at least 60-min post-prandial and before each football training session throughout spring camp and preseason football training, as previously described [1]. Briefly, HRV was measured for $1 \mathrm{~min}$ following a 1-min stabilization period in the seated position. HRV was derived from finger-pulse plethysmography and accompanying application (ithlete ${ }^{\mathrm{TM}}$, HRV Fit Ltd., Southampton, UK) on a tablet device, previously shown to provide acceptable agreement with electrocardiograph-derived HRV [20]. One-min HRV recordings obtained at the training facility in collision-sport athletes under similar conditions have demonstrated acceptable relative (Intraclass 
Correlation $=0.90)$ and absolute inter-day reliability $(\mathrm{CV}=7.65 \%)[16]$. After completion of the recording, the application automatically computes and displays the vagal-HRV parameter, the natural logarithm of the root mean square of successive differences (LnRMSSD), which is multiplied by twenty to fit an approximate 100-point scale. The application uses a previously described processing algorithm to filter artefacts and ectopic beats [1].

\subsubsection{Training Load}

External training load was quantified via $100 \mathrm{~Hz}$ tri-axial accelerometer (Catapult Innovations, Melbourne, Australia), fastened to the shoulder pads between the scapulae as previously described [1]. Total Player Load was selected for analysis because it reflects total external workload during training and is expressed as the square root of the sum of the squared instantaneous rate of change in acceleration in each vector and divided by 100 [21].

\subsubsection{Subjective Indicators of Wellbeing}

A daily wellness questionnaire adapted from McLean et al. [22] was completed following each HRV recording. The player rated his perceived level of sleep quality, fatigue, muscle soreness, stress, and mood on a 1-9 scale [23]. A rating of 5 represented feeling "okay" while greater and lower numbers reflected improvements or decrements, respectively, in a given parameter [23].

\subsubsection{Concussion Testing}

The cognitive assessment (Standardized Assessment of Concussion) and symptom scale of the second version of the Sport Concussion Assessment Tool (SCAT2) were used to document baseline status approximately 18 months prior to concussion occurrence. The corresponding components of the third version (SCAT3), which were unchanged in format from the previous versions, were used to document changes in symptom number, symptom severity, and cognitive function after concussion occurrence.

\subsection{Statistical Analysis}

LnRMSSD was analyzed by determining if daily values or the $\sim 7$-day rolling average positively or negatively exceeded the smallest worthwhile change (SWC). The SWC thresholds were calculated as \pm 0.5 of the coefficient of variation from the first seven measures obtained from spring camp and again from the first seven measures of preseason camp $[24,25]$. The mean (LnRMSSD $M$ ) and coefficient of variation (LnRMSSD $\mathrm{CV}_{\mathrm{CV}}$ ) from spring camp (13 recordings), Pre-SRC (9 recordings), and Post-SRC (11 recordings) were calculated. LnRMSSD values from scrimmage days were excluded due to potential effects of pre-competitive anxiety on autonomic activity (boxed data points in Figure 1). Pre-competition arousal can reduce HRV and therefore may obscure the typical day-to-day variation from training that we aimed to capture for comparison to post-SRC responses. Cohen's $d$ effect sizes (ES) $[26] \pm 90 \%$ confidence interval (CI) were used to compare LnRMSSD $_{M}$ and Player Load values between time-points using the following qualitative thresholds: $<0.2=$ trivial; $0.2-0.59=$ small; $0.6-1.19$ $=$ moderate $; 1.2-1.99=$ large $;$ and $>2.0$, very large [24]. All analyses were carried out using JMP Pro 12 (SAS Institute Inc., Cary, NC, USA) and Excel 2016 (Microsoft Office, Redmond, WA, USA). 

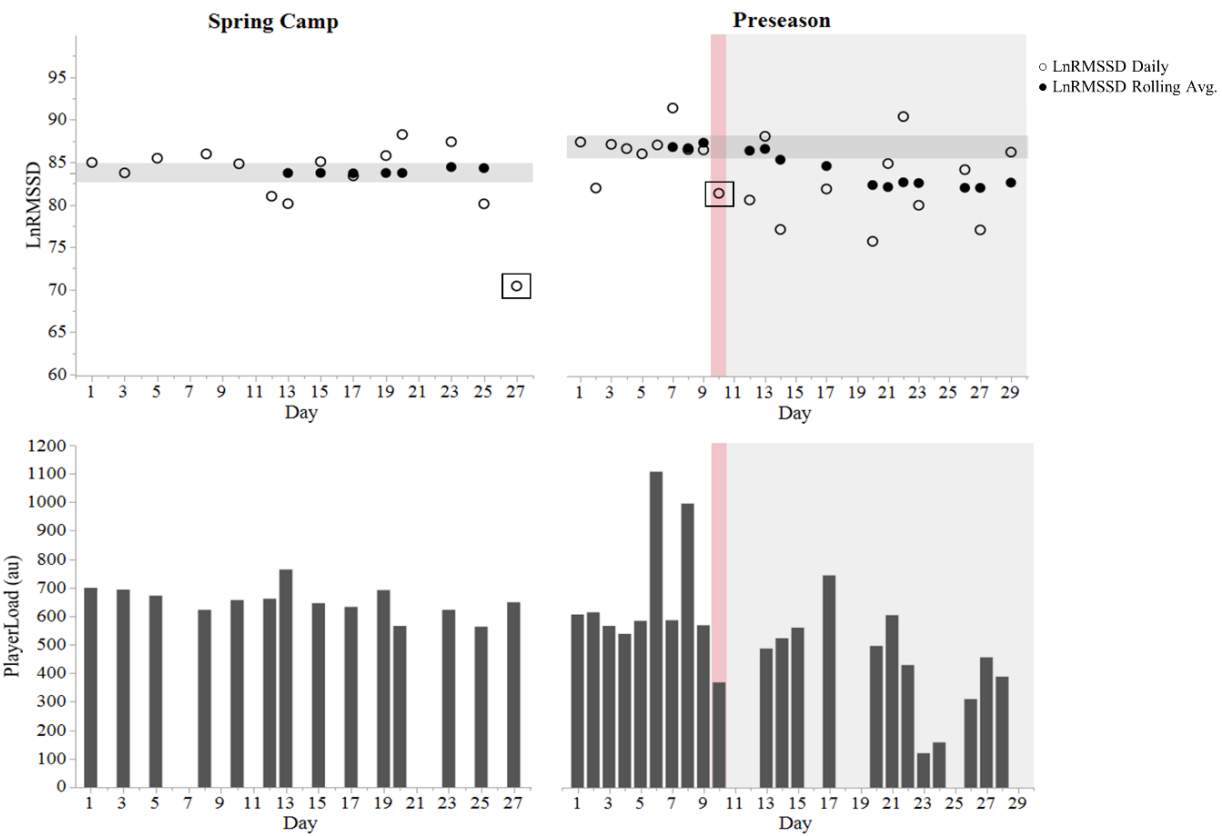

Figure 1. Natural logarithm of the root mean square of successive differences (LnRMSSD) and Player Load values throughout spring camp and preseason training. The horizontal shaded zone represents smallest worthwhile change thresholds for LnRMSSD. Red shade represents day of concussion. Grey shade represents the post-concussion period. Boxed data points represent scrimmage days.

\section{Results}

LnRMSSD and Player Load values from spring camp and preseason can be viewed in Figure 1. LnRMSSD $_{M}$ from spring camp, Pre-SRC, and Post-SRC were $84.3 \pm 2.6,86.8 \pm 2.4$, and $82.4 \pm 4.8$, respectively. Reductions in $\mathrm{LnRMSSD}_{\mathrm{M}}$ at Post-SRC were moderate (ES $=-1.12 \pm 0.80$ ) relative to

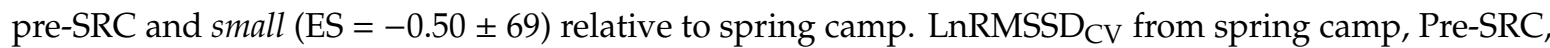
and Post-SRC was 3.1\%, $2.7 \%$, and 5.8\%, respectively.

Player Load values from spring camp, Pre-SRC, and Post-SRC were $655 \pm 54,542 \pm 66$, and $441 \pm 169$, respectively. Player Load from post-SRC was moderately lower than pre-SRC $(E S=-0.76 \pm$ $0.68)$ and largely lower than spring camp $(\mathrm{ES}=-1.73 \pm 0.74)$.

Decrements in some markers of well-being were reported within $48 \mathrm{~h}$ post-SRC but returned to pre-SRC ratings thereafter (Figure 2). Restricted (i.e., non-contact) football participation was performed as tolerated and without exacerbation of symptoms by $72 \mathrm{~h}$ post-SRC. Unrestricted football participation occurred at 7 days post-SRC. The athlete denied ever having sustained a concussion prior to the occurrence documented in this report. Concussion status indicators can be viewed in Table 1 .

Table 1. Change in indicators of status before and after concussion.

\begin{tabular}{cccc}
\hline Observation Date & Symptom Number $^{\mathbf{1}}$ & Symptom Severity $^{\mathbf{2}}$ & SAC Score $^{\mathbf{3}}$ \\
\hline Baseline & 0 & 0 & 27 \\
Day of Injury & 8 & 22 & 27 \\
Post-Injury Day 1 & 6 & 8 & - \\
Post-Injury Day 2 & 18 & 36 & - \\
Post-Injury Day 3 & 4 & 7 & 29 \\
Post-Injury Day 4 & - & - & - \\
Post-Injury Day 5 & 2 & 2 & - \\
Post-Injury Day 6 & 0 & 0 & 29 \\
\hline
\end{tabular}

\footnotetext{
${ }^{1}$ Sport Concussion Assessment Tool (maximum of 22); ${ }^{2}$ Sport Concussion Assessment Tool (maximum of 132);
}

${ }^{3}$ Standardized Assessment of Concussion (perfect score $=30$ ). 

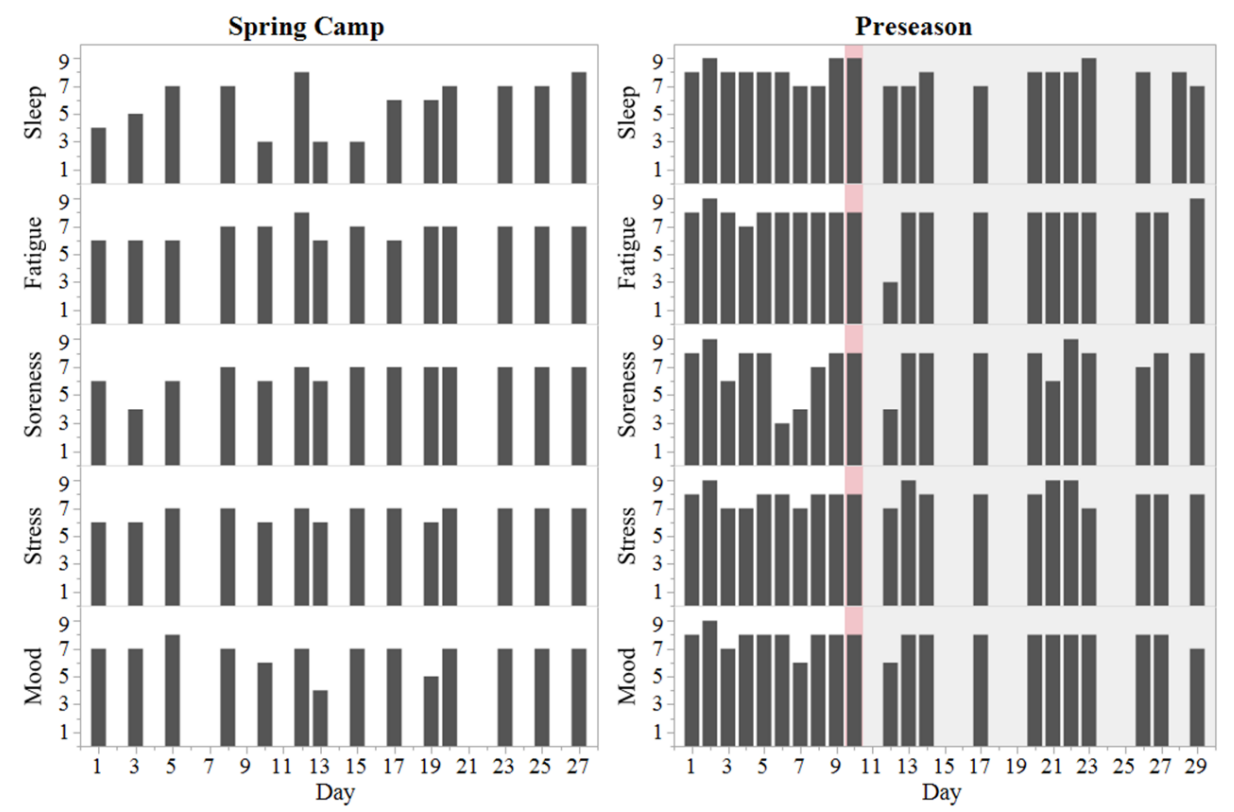

Figure 2. Subjective indicators of well-being throughout spring camp and preseason training. Red shade represents day of concussion (Day 10). Grey shade represents the post-concussion period.

\section{Discussion}

This case report documents alterations in serial measurements of HRV acquired from a college football player following SRC. A novel aspect of this report is the longitudinal observation period, enabling the establishment of baseline LnRMSSD trends to serve as a reference point for post-SRC data comparison. The main finding was that meaningful reductions in LnRMSSD persisted beyond return-to-play clearance and were graphically similar to trends exhibited by overreached athletes.

The athlete presented a remarkably low LnRMSSDcv during spring camp (3.1\%) and during the pre-SRC period (2.7\%). Average LnRMSSDcv values among players of the same position group during spring camp were $8.5 \pm 1.7 \%$ [1]. Low LnRMSSDcv generally reflects high fitness and positive adaptation to training among collision-sport athletes $[15,17]$. Conversely, an increased LnRMSSDcv

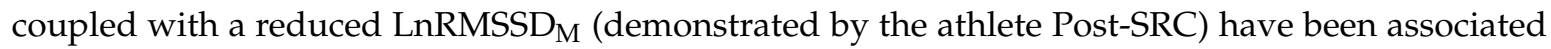
with high perceived fatigue and poor training adaptation $[17,18,23]$. Whether Post-SRC LnRMSSD changes were SRC- or training-induced is inconclusive due to the observational nature of this report. Peak Player Load values occurred on days 6 and 8 of the preseason (Pre-SRC) and represent two-a-day practice sessions (Figure 1). Cardiac-parasympathetic recovery from intensive training takes place within $24-48 \mathrm{~h}$ post-exercise [27]. In addition, highly-fit athletes have been shown to maintain stable LnRMSSD and subjective self-report measures of recovery despite significant $(p<0.05)$ increments in training load during preparatory training [15]. Therefore, reductions in LnRMSSD subsequent to day 10 (Post-SRC) were unlikely associated with the acute spikes in Player Load from days 6 and 8. In support of this postulation, Post-SRC Player Load values were moderately reduced relative to Pre-SRC, and subjective indicators were not dramatically altered (Figure 2), which generally facilitates the return of LnRMSSD to or above baseline [18]. Thus, we contend that the changes in LnRMSSD were likely SRC-induced rather than training fatigue-related.

Daily LnRMSSD was not chronically suppressed Post-SRC, but exhibited marked fluctuations (Figure 1). Consequently, the Pre-SRC vs. Post-SRC 90\% CI of the ES ranged from small-large. Reliance on single-time point HRV measures Post-SRC may therefore be insufficient and misleading to practitioners for assessing the effects of SRC on autonomic function. For example, if LnRMSSD was acquired in isolation on day $13,21,22,26$, or 29 , one might falsely conclude that SRC was no longer or only minimally affecting cardiac-autonomic activity. Whereas if LnRMSSD was obtained from any other day Post-SRC, converse interpretations would be made. Relative to isolated recordings, serial 
measures improved the diagnostic ability of LnRMSSD for identifying non-functional overreaching in a previous report [25]. More recently, coronary artery disease patients displayed greater daily fluctuations in self-recorded, home-based heart rate measures preceding a cardiovascular event relative to event-free patients who maintained a stable trend [28]. Though further research is needed to support the current observation of greater LnRMSSD fluctuations Post-SRC, it seems that detection of autonomic dysregulation Post-SRC may require near-daily assessment and that the magnitude of daily fluctuations (e.g., LnRMSSDcv) should be considered [15,18,25,28].

Autonomic dysregulation may be a readily measurable manifestation of the neurometabolic cascade that follows SRC $[29,30]$, but persisting dysfunction is probably mediated by diffuse axonal injury (DAI) that disrupts the structural connectivity within and between brain networks that regulate autonomic processes, emotional responses, and executive functions [12,31-33]. The brain's executive control network (ECN) and central autonomic network (CAN) are closely linked through bidirectional neural signals conveyed through common structural elements, including the anterior cingulate cortex [33,34]. Important internal or external stimuli are identified by the salience network (SN), which deactivates the internally-focused default mode network (DMN) and activates the ECN for a potential need to respond. This process releases the CAN "vagal brake" to prepare for the increased metabolic requirements of ECN engagement and responses to impending demands [34,35]. Dysfunctional network interactions can result in sympathetic hyperactivity in a resting state or during physical exertion. White matter tracts that often exhibit DAI include the genu of the corpus callosum, the anterior corona radiata, and the uncinate fasciculus [36,37]. Microstructural disruptions in these connections among components of the $\mathrm{SN}, \mathrm{ECN}, \mathrm{CAN}$, and rostral limbic system provide a plausible explanation for loss of normal regulatory control of attention, autonomic processes, and emotional responses [13]. Future research is needed to establish the extent to which HRV reflects neurological status following SRC and whether persistent decrements have relevance for clinical treatment or return to play considerations.

This report is the first to compare serial pre-injury and post-injury HRV measures in a college football player acquired with a method that is feasible for tracking the status of an entire roster of team-members [1,2]. Practitioners responsible for monitoring athletes should be aware that LnRMSSD responses to SRC appear similar to trends associated with stress and training fatigue, which may cause misinterpretation of an athlete's health and recovery status. Therefore, practitioners should maintain regular communication with the sports medicine staff when an athlete demonstrates alterations in their LnRMSSD trend, which could be due to an unreported SRC. Likewise, sports medicine staff should inform strength and conditioning coaches of head injuries experienced by players so that cardiac-autonomic function can be monitored and interpreted in the appropriate context. A potentially important limitation of our work is lack of HRV measurement in a state of low-level physical exertion, which may reveal persisting abnormalities that are not evident at rest $[10,12,13,38]$. Another limitation concerns the lack of a control subject. However, comparison of Post-SRC LnRMSSD to a longitudinal baseline may be superior to between-subject comparisons due to the individual nature of LnRMSSD trends, even among teammates of the same position [1]. For example, the LnRMSSDcv presented by the current athlete Post-SRC (5.8\%) would still be lower than most other players of the same position group [1] despite elevation from his longitudinal baseline $(\sim 3 \%)$. Finally, while efforts were made to control for location, environment, time of day, proximity to food or fluid intake, and physical activity, $\mathrm{HRV}$ is sensitive to a variety of stimuli [5] that may have contributed to daily variation in the measures.

\section{Conclusions}

This case report demonstrated sustained alterations in the daily HRV pattern of a college football player beyond return to play clearance from a SRC. The HRV responses to SRC appeared similar to trends associated with stress and training fatigue. Because accumulating evidence indicates that SRC can have persisting adverse effects, serial HRV measurements may provide a clinically feasible means to objectively track recovery. In addition to fatigue and recovery status information, the convenient and 
time-efficient HRV monitoring procedures used in this case report may also be sensitive to physiological responses to SRC that may be relevant for clinical treatment and return to play considerations.

Author Contributions: Conceptualization, A.A.F.; Formal analysis, A.A.F.; Investigation, A.A.F., J.R.A. and C.M.K.; Methodology, A.A.F. and J.R.A.; Supervision, M.R.E.; Writing—original draft, A.A.F. and G.B.W.; Writing一review and editing, M.R.E., J.R.A., C.M.K.

Funding: No funding was received for this study.

Acknowledgments: We thank the player and team-staff members for their involvement in this study.

Conflicts of Interest: The authors have no conflicts of interest to report.

\section{References}

1. Flatt, A.A.; Esco, M.R.; Allen, J.R.; Robinson, J.B.; Earley, R.L.; Fedewa, M.V.; Bragg, A.; Keith, C.M.; Wingo, J.E. Heart rate variability and training load among national collegiate athletic association division 1 college football players throughout spring camp. J. Strength. Cond. Res. 2017, 32, 3127-3134. [CrossRef] [PubMed]

2. Flatt, A.A.; Esco, M.R.; Allen, J.R.; Robinson, J.B.; Bragg, A.; Keith, C.M.; Fedewa, M.V.; Earley, R.L. Cardiac-autonomic responses to in-season training among division-1 college football players. J. Strength. Cond. Res. 2019. [CrossRef] [PubMed]

3. Wellman, A.D.; Coad, S.C.; Flynn, P.J.; Climstein, M.; McLellan, C.P. Movement demands and perceived wellness associated with preseason training camp in NCAA division I college football players. J. Strength. Cond. Res. 2017, 31, 2704-2718. [CrossRef] [PubMed]

4. Fullagar, H.H.; Govus, A.; Hanisch, J.; Murray, A. The time course of perceptual recovery markers after match play in division ia college American football. Int. J. Sports Physiol. Perf. 2017, 12, 1264-1266. [CrossRef]

5. Laborde, S.; Mosley, E.; Mertgen, A. A unifying conceptual framework of factors associated to cardiac vagal control. Heliyon 2018, 4, e01002. [CrossRef]

6. Dompier, T.P.; Kerr, Z.Y.; Marshall, S.W.; Hainline, B.; Snook, E.M.; Hayden, R.; Simon, J.E. Incidence of concussion during practice and games in youth, high school, and collegiate American football players. JAMA Pediat. 2015, 169, 659-665. [CrossRef] [PubMed]

7. De Beaumont, L.; Theoret, H.; Mongeon, D.; Messier, J.; Leclerc, S.; Tremblay, S.; Ellemberg, D.; Lassonde, M. Brain function decline in healthy retired athletes who sustained their last sports concussion in early adulthood. Brain 2009, 132, 695-708. [CrossRef]

8. De Beaumont, L.; Lassonde, M.; Leclerc, S.; Théoret, H. Long-term and cumulative effects of sports concussion on motor cortex inhibition. Neurosurgery 2007, 61, 329-337. [CrossRef]

9. Ellis, M.J.; Leddy, J.J.; Willer, B. Physiological, vestibulo-ocular and cervicogenic post-concussion disorders: An evidence-based classification system with directions for treatment. Brain Inj. 2015, 29, 238-248. [CrossRef]

10. Senthinathan, A.; Mainwaring, L.M.; Hutchison, M. Heart rate variability of athletes across concussion recovery milestones: A preliminary study. Clin. J. Sport Med. 2017, 27, 288-295. [CrossRef] [PubMed]

11. Maruta, J.; Lee, S.W.; Jacobs, E.F.; Ghajar, J. A unified science of concussion. Ann N Y Acad. Sci. 2010, 1208, 58-66. [CrossRef]

12. Abaji, J.P.; Curnier, D.; Moore, R.D.; Ellemberg, D. Persisting effects of concussion on heart rate variability during physical exertion. J. Neurotrauma 2016, 33, 811-817. [CrossRef]

13. Conder, R.L.; Conder, A.A. Heart rate variability interventions for concussion and rehabilitation. Front. Psychol. 2014, 5, 890. [CrossRef]

14. Flatt, A.A.; Howells, D.; Williams, S. Effects of consecutive domestic and international tournaments on heart rate variability in an elite rugby sevens team. J. Sci. Med. Sport 2019, 22, 616-621. [CrossRef]

15. Flatt, A.A.; Howells, D. Effects of varying training load on heart rate variability and running performance among an Olympic rugby sevens team. J. Sci. Med. Sport 2018, 22, 222-226. [CrossRef] [PubMed]

16. Nakamura, F.Y.; Pereira, L.A.; Esco, M.R.; Flatt, A.A.; Moraes, J.E.; Abad, C.C.C.; Loturco, I. Intraday and interday reliability of ultra-short-term heart rate variability in rugby union players. J. Strength. Cond. Res. 2017, 31, 548-551.

17. Flatt, A.A.; Esco, M.R. Evaluating individual training adaptation with smartphone-derived heart rate variability in a collegiate female soccer team. J. Strength. Cond. Res. 2016, 30, 378-385. [CrossRef] 
18. Flatt, A.A.; Hornikel, B.; Esco, M.R. Heart rate variability and psychometric responses to overload and tapering in collegiate sprint-swimmers. J. Sci. Med. Sport. 2017, 20, 606-610. [CrossRef]

19. Reed, N.; Murphy, J.; Dick, T.; Mah, K.; Paniccia, M.; Verweel, L.; Dobney, D.; Keightley, M. A multi-modal approach to assessing recovery in youth athletes following concussion. JoVE 2014, 91, e51892. [CrossRef]

20. Esco, M.R.; Flatt, A.A.; Nakamura, F.Y. Agreement between a smart-phone pulse sensor application and ecg for determining lnrmssd. J. Strength. Cond. Res. 2016, 31, 380-385. [CrossRef]

21. Boyd, L.J.; Ball, K.; Aughey, R.J. The reliability of minimaxx accelerometers for measuring physical activity in Australian football. Int. J. Sports Physiol. Perf. 2011, 6, 311-321. [CrossRef]

22. McLean, B.D.; Coutts, A.J.; Kelly, V.; McGuigan, M.R.; Cormack, S.J. Neuromuscular, endocrine, and perceptual fatigue responses during different length between-match microcycles in professional rugby league players. Int. J. Sports Physiol. Perf. 2010, 5, 367-383. [CrossRef]

23. Flatt, A.A.; Esco, M.R.; Nakamura, F.Y. Association between subjective indicators of recovery status and heart rate variability among divison-1 sprint-swimmers. Sports 2018, 6, 93. [CrossRef]

24. Hopkins, W.; Marshall, S.; Batterham, A.; Hanin, J. Progressive statistics for studies in sports medicine and exercise science. Med. Sci. Sports Exerc. 2009, 41, 3-13. [CrossRef]

25. Plews, D.J.; Laursen, P.B.; Kilding, A.E.; Buchheit, M. Heart rate variability in elite triathletes, is variation in variability the key to effective training? A case comparison. Eur. J. Appl. Physiol. 2012, 112, 3729-3741. [CrossRef] [PubMed]

26. Cohen, J. Statistical Power Analysis for the Behavioral Sciences, 2nd ed.; Routledge: Hillsdale, NJ, USA, 1988.

27. Stanley, J.; Peake, J.M.; Buchheit, M. Cardiac parasympathetic reactivation following exercise: Implications for training prescription. Sports Med. 2013, 43, 1259-1277. [CrossRef]

28. Tulppo, M.P.; Kiviniemi, A.M.; Junttila, M.J.; Huikuri, H.V. Home monitoring of heart rate as a predictor of imminent cardiovascular events. Front. Physiol. 2019, 10, 341. [CrossRef] [PubMed]

29. La Fountaine, M.F. An anatomical and physiological basis for the cardiovascular autonomic nervous system consequences of sport-related brain injury. Int. J. Psychophysiol. 2017, 132, 155-166. [CrossRef] [PubMed]

30. Leddy, J.; Hinds, A.; Sirica, D.; Willer, B. The role of controlled exercise in concussion management. PMR 2016, 8, S91-S100. [CrossRef] [PubMed]

31. Sharp, D.J.; Beckmann, C.F.; Greenwood, R.; Kinnunen, K.M.; Bonnelle, V.; De Boissezon, X.; Powell, J.H.; Counsell, S.J.; Patel, M.C.; Leech, R. Default mode network functional and structural connectivity after traumatic brain injury. Brain 2011, 134, 2233-2247. [CrossRef] [PubMed]

32. Thayer, J.F.; Hansen, A.L.; Saus-Rose, E.; Johnsen, B.H. Heart rate variability, prefrontal neural function, and cognitive performance: The neurovisceral integration perspective on self-regulation, adaptation, and health. Ann. Behav. Med. 2009, 37, 141-153. [CrossRef]

33. Winkelmann, T.; Thayer, J.F.; Pohlack, S.; Nees, F.; Grimm, O.; Flor, H. Structural brain correlates of heart rate variability in a healthy young adult population. Brain Struct. Funct. 2017, 222, 1061-1068. [CrossRef] [PubMed]

34. Jennings, J.R.; Sheu, L.K.; Kuan, D.C.H.; Manuck, S.B.; Gianaros, P.J. Resting state connectivity of the medial prefrontal cortex covaries with individual differences in high-frequency heart rate variability. Psychophysiology 2016, 53, 444-454. [CrossRef] [PubMed]

35. Holzman, J.B.; Bridgett, D.J. Heart rate variability indices as bio-markers of top-down self-regulatory mechanisms: A meta-analytic review. Neurosci. Biobehav. Rev. 2017, 74, 233-255. [CrossRef] [PubMed]

36. Niogi, S.; Mukherjee, P.; Ghajar, J.; Johnson, C.; Kolster, R.; Sarkar, R.; Lee, H.; Meeker, M.; Zimmerman, R.; Manley, G. Extent of microstructural white matter injury in postconcussive syndrome correlates with impaired cognitive reaction time: A 3t diffusion tensor imaging study of mild traumatic brain injury. Am. J. Neuroradiol. 2008, 29, 967-973. [CrossRef] [PubMed]

37. Maruta, J.; Suh, M.; Niogi, S.N.; Mukherjee, P.; Ghajar, J. Visual tracking synchronization as a metric for concussion screening. J. Head. Trauma. Rehabil. 2010, 25, 293-305. [CrossRef]

38. La Fountaine, M.F.; Gossett, J.D.; De Meersman, R.E.; Bauman, W.A. Increased qt interval variability in 3 recently concussed athletes: An exploratory observation. J. Athl. Train. 2011, 46, 230-233. [CrossRef]

(C) 2019 by the authors. Licensee MDPI, Basel, Switzerland. This article is an open access article distributed under the terms and conditions of the Creative Commons Attribution (CC BY) license (http://creativecommons.org/licenses/by/4.0/). 